\title{
Erythrocyte transfusion limits the role of elevated red cell distribution width on predicting cardiac surgery associated acute kidney injury
}

Wuhua Jiang, 2,3*, Zhouping Zou ${ }^{4 *}$, Shuan Zhao ${ }^{1,2,3 *}$, Yi Fang ${ }^{1,2,3}$, Jiarui Xu ${ }^{1,2,3}$, Yimei Wang ${ }^{1,2,3}$, Bo Shen ${ }^{1,2,3}$, Zhe Luo ${ }^{5}$, Chunsheng Wang ${ }^{6}$, Xiaoqiang Ding $^{1,2,3,7}$, Jie Teng ${ }^{1,2,3,7}$

${ }^{1}$ Department of Nephrology, Zhongshan Hospital, Fudan University, Shanghai, China

${ }^{2}$ Shanghai Medical Center of Kidney, Shanghai, China

${ }^{3}$ Shanghai Key Laboratory of Kidney and Blood Purification, Shanghai, China

${ }^{4}$ Department of Nephrology, Xuhui Zhongshan Hospital, Fudan University, Shanghai, China ${ }^{5}$ Department of Intensive Care Medicine, Zhongshan Hospital, Fudan University, Shanghai, China

${ }^{6}$ Department of Cardiac Surgery, Zhongshan Hospital, Fudan University, Shanghai, China ${ }^{7}$ Department of Nephrology, Xiamen Branch, Zhongshan Hospital, Fudan University, Xiamen, China

\begin{abstract}
Background: Acute kidney injury (AKI) is one of the more serious complications after cardiac surgery. Elevated red cell distribution width (RDW) was reported as a predictor for cardiac surgery associated acute kidney injury (CSAKI). However, the increment of RDW by erythrocyte transfusion makes its prognostic role doubtful. The aim of this study is to elucidate the impact of erythrocyte transfusion on the prognostic role of elevated RDW for predicting CSAKI.

Methods: A total of 3207 eligible patients who underwent cardiac surgery during 2016-2017 were enrolled. Changes of $R D W$ was defined as the difference between preoperative $R D W$ and $R D W$ measured $24 \mathrm{~h}$ after cardiac surgery. The primary outcome was CSAKI which was defined by the Kidney Disease: Improving Global Outcomes Definition and Staging (KDIGO) criteria. Univariate and multivariate analysis were performed to identify predictors for CSAKI.

Results: The incidence of CSAKI was $38.07 \%$ and the mortality was $1.18 \%$. CSAKI patients had higher elevated $R D W$ than those without CSAKI (0.65\% vs. $0.39 \%, p<0.001)$. Multivariate regression showed that male, age, New York Heat Association classification 3-4, elevated RDW, estimated glomerular filtration rate $<60 \mathrm{~mL} / \mathrm{min} / 1.73 \mathrm{~m}^{2}$, cardiopulmonary bypass time $>120 \mathrm{~min}$ and erythrocyte transfusion were associated with CSAKI. Subgroup analysis showed elevated RDW was an independent predictor for CSAKI in the non-transfused subset (adjusted odds ratio: 1.616, $p<0.001$ ) whereas no significant association between elevated RDW and CSAKI was found in the transfused patients (odds ratio: $1.040, p=0.497$ ).
\end{abstract}

Conclusions: Elevated RDW is one of the independent predictors of CSAKI in the absence of erythrocyte transfusion, which limits the prognostic role of the former on predicting CSAKI. (Cardiol J 2021; 28, 2: 255-261)

Key words: red cell distribution width, erythrocyte transfusion, cardiac surgery, acute kidney injury

Address for correspondence: Dr. Jie Teng, Department of Nephrology, Zhongshan Hospital, Fudan University,

No. 180 Fenglin Road, Shanghai 200032, China, tel: +86 2164041990 2439, fax: +86 2164223006 ,

e-mail: teng.jie@zsxmhospital.com

Received: 26.11.2019 Accepted: 21.04.2020

*Wuhua Jiang, Zhouping Zou, Shuan Zhao contributed equally for this work.

This article is available in open access under Creative Common Attribution-Non-Commercial-No Derivatives 4.0 International (CC BY-NC-ND 4.0) license, allowing to download articles and share them with others as long as they credit the authors and the publisher, but without permission to change them in any way or use them commercially. 


\section{Introduction}

Acute kidney injury (AKI) is one of the prevalent and severe complications after cardiac surgery. The mortality of patients who develop cardiac surgery associated acute kidney injury (CSAKI) or severe AKI with renal replacement therapy (RRT) required remains high [1]. The diagnosis of AKI is mainly based on serum creatine and urine output, whereas these functional markers are insufficient to predict AKI at an earlier stage. Therefore, new biomarkers have been studied for diagnosing AKI earlier [2-5]. Although new biomarkers for predicting AKI have been developed, they are expensive, and restrain the prevalence of utilization in developing countries like India, Brazil and the Chinese mainland, where cardiac surgery is booming and AKI incidence is high.

Red cell distribution width (RDW) is recognized as an index of erythrocyte volume variability and is routinely reported as a part of a complete blood cell count. Recently, its role of predicting CSAKI has been revealed [6, 7]. RDW is reported associating with inflammation or ischemia reperfusion injury $[8,9]$, and is likely to elevate after cardiac surgery, especially surgery with cardiopulmonary bypass (CPB) [10]. Accordingly, postoperative elevated RDW is potentially associated with the oxidative stress and inflammation during operation and early phase of the postoperative period.

Meanwhile, erythrocyte transfusion is performed widely in cardiac surgery and reported as a potentially modifiable risk factor for CSAKI [11]. Recent evidence suggests that RDW increases after erythrocyte transfusion [12]. However, little is known about whether the increment of RDW by transfusion will influence its value for predicting CSAKI.

The purpose of the present study is to validate the role of elevated RDW predicting CSAKI and analyze whether or not its prognostic role is confounded by erythrocyte transfusion.

\section{Methods}

\section{Patient sample}

This study was approved by the ethical board of Zhongshan Hospital, Fudan University (Approval Number B2017-039). Informed consent was obtained from all participants. Data from consecutive patients aged 18 years or older who underwent valve and/or coronary artery bypass grafting (CABG) surgery from January 2016 to December 2017 were included in this single-center cohort study. To reduce the confounding effect of acute life-threatening blood loss, patients who received plasma, platelet or more than four units of erythrocytes on the day of surgery were excluded. Other exclusion criteria were: transfusion of red blood cell (RBC) during the 28 days before enrollment, urgent surgery, preoperative mechanical ventilation or tracheotomy, preoperative defibrillator or ventricular assist devices, preoperative RRT, preoperative liver dysfunction, or sepsis.

\section{Data collection}

All perioperative data were prospectively collected and extracted retrospectively from the database of Zhongshan Hospital cardiac surgery. All data were checked twice by professional personnel before input into the database. Demographic and procedure-related variables known to be associated with AKI were included in this study after a literature review. They included gender, age, comorbidities, contrast media exposure history, preoperative cardiac function status (New York heart association [NYHA] classification), baseline estimated glomerular filtration rate (eGFR, calculated with CKD-EPI formulae [13]), surgical type, $\mathrm{CPB}$ duration, and erythrocyte transfusion amount on the day of surgery. Each unit of erythrocyte contains $300 \mathrm{~mL}$. Full blood counts were measured from BD EDTA-K2 samples using a Sysmex XN9000 electronic counter. Both preoperative and post-operative RDW were collected and changes of RDW was defined as the difference between preoperative RDW and RDW measured $24 \mathrm{~h}$ after cardiac surgery. The reference range of $\mathrm{RDW}$ value was $11.0-16.0 \%$ in this hospital. If there were more than one cardiac surgery procedures performed during a single hospitalization, only the data on the first surgery was included in the analysis.

The primary end-point was postoperative AKI. AKI was defined according to the KDIGO guideline [14] as any of the following: increase in $\mathrm{SCr}$ by $\geq 0.3 \mathrm{mg} / \mathrm{dL}(\geq 26.5 \mu \mathrm{mol} / \mathrm{L})$ within $48 \mathrm{~h}$; or increase in $\mathrm{SCr}$ to $\geq 1.5$ times the baseline that is known or presumed to have occurred within the prior 7 days or urine volume $<0.5 \mathrm{~mL} / \mathrm{kg} / \mathrm{h}$ for $6 \mathrm{~h}$.

\section{Statistical analyses}

Statistical analyses were performed by SPSS statistics for Windows (Version 25.0. IBM Corp, Armonk, NY). Continuous variables were expressed as the mean \pm standard deviation $(\mathrm{SD})$ and analyzed by unpaired t-tests, with the Welch adjustment when necessary. Continuous variables that violated the normality assumption were expressed 
as median and $25^{\text {th }}$ to $75^{\text {th }}$ percentiles and analyzed by the Mann-Whitney U test. Categorical variables were expressed as absolute (n) and relative (\%) frequency and were analyzed by the Pearson 2-test or the Fisher exact test whenever appropriate. A significant level was considered $\mathrm{p}<0.05$.

Univariate analyses were performed to identify a potential association with CSAKI and those with $\mathrm{p}<0.05$ were entered into multivariate regression analysis to identify independent risk factors for both end-points. An adjusted logistic regression model was developed with variables that showed $\mathrm{p}<0.05$ in univariate analysis.

Subgroup analysis was performed to elucidate the impact of erythrocyte transfusion on the prognostic role of elevated RDW for CSAKI. Patients were classified into two groups according to whether receiving transfusions or not. Multivariable regressions were performed to identify the predictive role of elevated RDW for CSAKI in both subsets.

\section{Results}

\section{Baseline characteristics}

A total of 3207 eligible patients who underwent cardiac surgery during 2016-2017 were enrolled in this cohort study. Characteristics of patients are presented in Table 1 . The CSAKI rate in the entire cohort was $38.07 \%$ (1221/3207). Among AKI patients, the incidence of stage 1, 2 and 3 were $72.9 \%(890 / 1221), 17.5 \%$ (214/1221) and $9.6 \%$ (117/1221). Male, elder, and those who had more comorbidities such as hypertension and impaired preoperative cardiac and renal function were likely to develop CSAKI. Those patients who underwent complex surgery with multiple procedures or CPB were more inclined to develop CSAKI as well. However, patients in the present study were classified as undergoing valve surgery and no significant relation was found between the occurrence of CSAKI and multidirectional surgery types. The postoperative RDW and elevated RDW were higher in patients who developed CSAKI. Moreover, AKI patients received more RBC transfusions. The in-hospital mortality ( 2.8 vs. $0.2 \%$, $\mathrm{p}<0.001)$ and length of stay (14 vs. $13 \%$, $\mathrm{p}<0.001)$ of CSAKI patients were significantly higher as well (Table 1). The magnitude of elevated RDW were higher in patients who received RBC transfusions, regardless of the occurrence of AKI. However, no significant trend of elevated RDW between different $\mathrm{RBC}$ transfusion groups was found (Fig. 1).

\section{Predictors for CSAKI}

Univariate analysis was performed to identify potential risk factors associated with CSAKI from the variables that showed a $\mathrm{p}$ value $<0.05$ in Table 1. Male gender (odds ratio [OR]:1.596, 95\% CI 1.375-1.852), age (per year) (OR: $1.030,95 \%$ CI 1.024-1.037), preoperative hypertension (OR: $1.288,95 \%$ CI 1.108-1.497), NYHA classification 3-4 (OR: 1.407, 95\% CI 1.215-1.629), preoperative eGFR < $60 \mathrm{~mL} / \mathrm{min} / 1.73 \mathrm{~m}^{2}$ (OR: $2.399,95 \%$ CI 1.851-3.108), complex procedure (OR: $2.752,95 \%$ CI 2.001-3.784), CPB time > 120 min (OR: 2.134, 95\% CI 1.752-2.599), erythrocyte transfusion (per unit) (OR: 1.340, 95\% CI 1.243-1.445) and elevated RDW (OR: 1.335, 95\% CI 1.232-1.447) were identified as potential predictors for CSAKI (Table 2).

Multivariate regression was developed with variables that showed a $\mathrm{p}<0.05$ in the univariate analysis. Male gender (OR: $2.127,95 \% \mathrm{CI}$ 1.759-2.571), age (per year) (OR: $1.033,95 \% \mathrm{CI}$ 1.024-1.042), NYHA classification 3-4 (OR: 1.214, 95\% CI 1.002-1.471), preoperative eGFR $<60 \mathrm{~mL} /$ $/ \mathrm{min} / 1.73 \mathrm{~m}^{2}$ (OR: $1.602,95 \%$ CI 1.124-2.284), CPB time $>120$ min (OR: 1.919, 95\% CI 1.553-2.372), erythrocyte transfusion (per unit) (OR: $1.167,95 \%$ CI 1.056-1.289) and elevated RDW (OR: 1.108, 95\% CI 1.005-1.222) were identified as independent predictors for CSAKI (Table 2).

\section{Subgroup analysis}

Patients were classified into two groups by whether they received transfusions or not. The CSAKI rate of the transfused patients was higher than the non-transfused $(46.9 \%$ vs. $33.8 \%, \mathrm{p}<0.001)$.

Multivariate regression was performed in both subgroups and showed elevated RDW was associated with CSAKI (OR: 1.613, p < 0.001) in the non-transfused group whereas no significance between the elevated RDW and CSAKI $(\mathrm{p}=0.497)$ was found in the transfused subgroup (Table 3 ).

The proportion of valve surgery is higher in Chinese patients undergoing heart surgery. A subgroup analysis was performed of patients undergoing valve surgery finding no significant correlation between e-RDW and CSAKI in patients undergoing blood transfusion, while increased e-RDW was a risk factor for CSAKI in non-transfused patients (OR: 1.877) (Table 4).

\section{Discussion}

The current study found that patients who developed CSAKI were more male, elderly and 
Table 1. Perioperative patient characteristics of the study population.

\begin{tabular}{|c|c|c|c|}
\hline Characteristics & Without AKI (n = 1986) & $A K I(n=1221)$ & $\mathbf{P}$ \\
\hline \multicolumn{4}{|l|}{ Demographic data } \\
\hline Male & $1121(56.4 \%)$ & $823(67.4 \%)$ & $<0.001$ \\
\hline Age [years] & $55.21 \pm 12.68$ & $59.22 \pm 10.92$ & $<0.001$ \\
\hline \multicolumn{4}{|l|}{ Medical history } \\
\hline Hypertension & $619(31.2 \%)$ & $450(36.9 \%)$ & $<0.001$ \\
\hline Diabetes mellitus & $214(10.8 \%)$ & $148(12.1 \%)$ & 0.244 \\
\hline NYHA classification 3-4 & $1108(55.8 \%)$ & $781(64.0 \%)$ & $<0.001$ \\
\hline \multicolumn{4}{|l|}{ Laboratory index } \\
\hline Hemoglobin $[\mathrm{g} / \mathrm{L}]$ & $133.54 \pm 14.98$ & $134 \pm 15.99$ & 0.419 \\
\hline Albumin $[\mathrm{g} / \mathrm{L}]$ & $39.83 \pm 3.10$ & $39.62 \pm 3.21$ & 0.071 \\
\hline Pre-op RDW [\%] & $13.43 \pm 1.32$ & $13.48 \pm 1.28$ & 0.187 \\
\hline Post-op RDW [\%] & $13.79 \pm 1.60$ & $14.13 \pm 1.55$ & $<0.001$ \\
\hline Elevated RDW [\%] & $0.39 \pm 0.22$ & $0.65 \pm 0.23$ & $<0.001$ \\
\hline \multicolumn{4}{|l|}{ Kidney function } \\
\hline Serum creatinine $[\mu \mathrm{mol} / \mathrm{L}]$ & $76.84 \pm 19.31$ & $84.83 \pm 31$ & $<0.001$ \\
\hline eGFR $\left[\mathrm{mL} / \mathrm{min} / 1.73 \mathrm{~m}^{2}\right]$ & $90.99 \pm 21.42$ & $84.86 \pm 22.98$ & $<0.001$ \\
\hline \multicolumn{4}{|l|}{ Procedure } \\
\hline Isolated valve & $1271(64.0 \%)$ & $717(58.7 \%)$ & 0.003 \\
\hline \multicolumn{4}{|l|}{ Single valve surgery: } \\
\hline AVR & $374(18.83 \%)$ & $158(12.94 \%)$ & 0.106 \\
\hline MVP & $187(9.41 \%)$ & $120(9.82 \%)$ & 0.981 \\
\hline MVR & $148(7.45 \%)$ & $146(11.95 \%)$ & 0.225 \\
\hline \multicolumn{4}{|l|}{ Double valve surgery: } \\
\hline $\mathrm{AVR}+\mathrm{MVP}$ & $11(0.56 \%)$ & $35(2.86 \%)$ & 0.078 \\
\hline DVR & $146(7.35 \%)$ & $69(5.65 \%)$ & 0.498 \\
\hline MVR+TVP & $157(7.90 \%)$ & $65(5.32 \%)$ & 0.497 \\
\hline \multicolumn{4}{|l|}{ Triple valve surgery: } \\
\hline $\mathrm{AVR}+\mathrm{MVR}+\mathrm{TVP}$ & $110(5.53 \%)$ & $104(8.50 \%)$ & 0.355 \\
\hline Others* & $138(6.94 \%)$ & $20(1.63 \%)$ & 0.588 \\
\hline Minimal invasive valve surgery & $128(6.44 \%)$ & $72(5.89 \%)$ & 0.974 \\
\hline Isolated CABG & $650(32.7 \%)$ & $400(32.8 \%)$ & 0.986 \\
\hline Valve and CABG & $65(3.3 \%)$ & $104(8.5 \%)$ & $<0.001$ \\
\hline CPB time [min] & $93.61 \pm 30.58$ & $110.82 \pm 36.39$ & $<0.001$ \\
\hline \multicolumn{4}{|l|}{ Erythrocyte transfusion** } \\
\hline $0 U$ & $1427(71.9 \%)$ & $728(59.6 \%)$ & $<0.001$ \\
\hline $1 U$ & $397(20.0 \%)$ & $303(24.8 \%)$ & 0.001 \\
\hline $2 U$ & $39(2.0 \%)$ & $52(4.3 \%)$ & $<0.001$ \\
\hline $3 U$ & $107(5.4 \%)$ & $105(8.6 \%)$ & $<0.001$ \\
\hline $4 U$ & $16(0.8 \%)$ & $33(2.7 \%)$ & $<0.001$ \\
\hline \multicolumn{4}{|l|}{ Prognosis } \\
\hline In-hospital mortality & $4(0.2 \%)$ & $34(2.8 \%)$ & $<0.001$ \\
\hline Length of hospital stay & $13(10-16)$ & $14(11-18)$ & $<0.001$ \\
\hline
\end{tabular}

The values are expressed as the median (interquartile range) and mean \pm standard deviation or number (percentage). $\mathrm{P}$-values are the results of unpaired t-test or Mann-Whitney $U$ test for continuous variables, and $\chi^{2}$ test or Fisher's exact test for categorical variables.

*Other procedures include tricuspid valve surgery, repairment of paraprosthetic regurgitation.

**The amount of erythrocyte transfusion refers to the total amount of erythrocyte transfusion for each patient received on the day of surgery. $\mathrm{AKI}$ - acute kidney injury; AVR - aortic valve replacement; CABG - coronary artery bypass grafting; CPB - cardiopulmonary bypass;

DVR - aortic valve replacement and mitral valve replacement; eGFR - estimated glomerular filtration rate, calculated by CKD-EPI formulae;

MVP — mitral valve plasty; MVR - mitral valve replacement; NYHA - New York Heart Association; RDW - red cell distribution width;

TVP - tricuspid valve plasty 


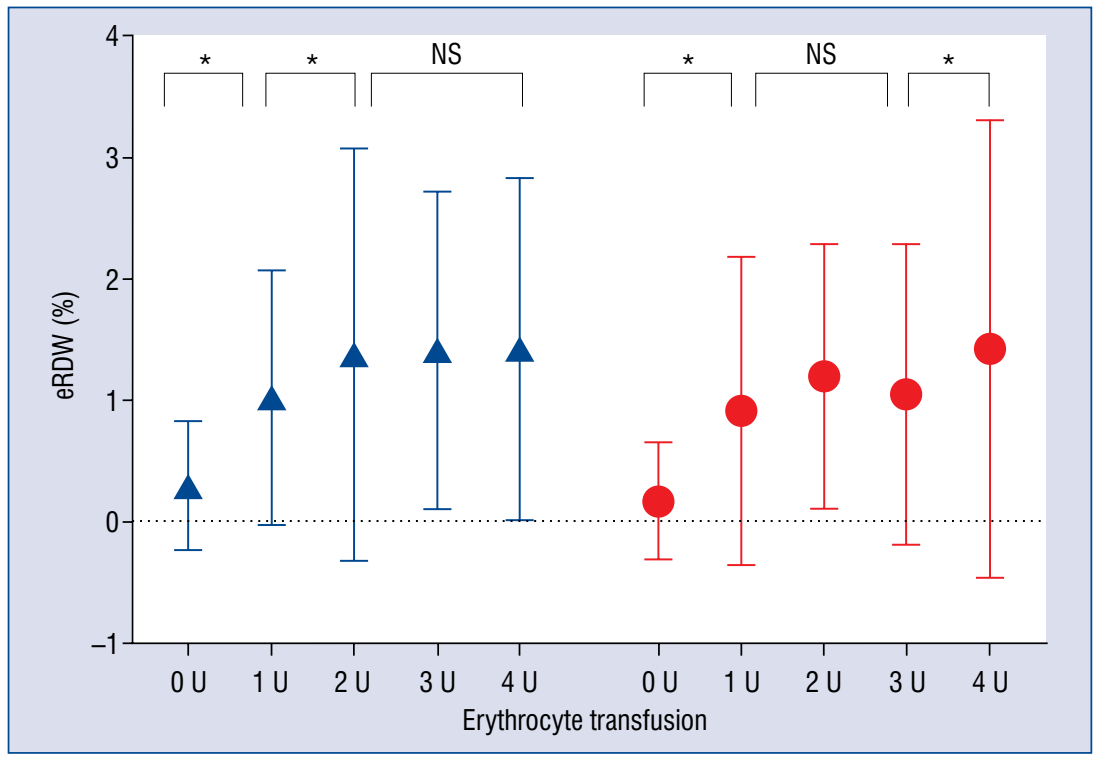

Figure 1. The magnitude of elevated red cell distribution width (eRDW) between different transfusion amount in both acute kidney injury (AKI) and non-AKI subgroups; ${ }^{*} \mathrm{p}<0.001$; NS - not significant; $\triangle$ - AKI; - NONAKI.

Table 2. Analysis of risk factors for CSAKI in entire cohort.

\begin{tabular}{|c|c|c|c|c|c|c|}
\hline \multirow[t]{2}{*}{ Variables } & \multicolumn{3}{|c|}{ Unadjusted } & \multicolumn{3}{|c|}{ Adjusted } \\
\hline & OR & $95 \% \mathrm{Cl}$ & $\mathbf{P}$ & OR & $95 \% \mathrm{Cl}$ & $\mathbf{P}$ \\
\hline Male & 1.596 & $1.375-1.852$ & $<0.001$ & 2.127 & $1.759-2.571$ & $<0.001$ \\
\hline Age [years] & 1.030 & $1.024-1.037$ & $<0.001$ & 1.033 & $1.024-1.042$ & $<0.001$ \\
\hline Hypertension & 1.288 & $1.108-1.497$ & $<0.001$ & & & \\
\hline NYHA classification 3-4 & 1.407 & $1.215-1.629$ & $<0.001$ & 1.214 & $1.002-1.471$ & 0.048 \\
\hline Elevated RDW (\%) & 1.335 & $1.232-1.447$ & $<0.001$ & 1.108 & $1.005-1.222$ & 0.039 \\
\hline eGFR $<60 \mathrm{~mL} / \mathrm{min} / 1.73 \mathrm{~m}^{2}$ & 2.399 & $1.851-3.108$ & $<0.001$ & 1.602 & $1.124-2.284$ & 0.009 \\
\hline Valve + CABG & 2.752 & $2.001-3.784$ & $<0.001$ & & & \\
\hline CPB time $>120 \mathrm{~min}$ & 2.134 & $1.752-2.599$ & $<0.001$ & 1.919 & $1.553-2.372$ & $<0.001$ \\
\hline Erythrocyte transfusion [U]* & 1.340 & $1.243-1.445$ & $<0.001$ & 1.167 & $1.056-1.289$ & 0.002 \\
\hline
\end{tabular}

*The amount of erythrocyte transfusion refers to the total amount of erythrocyte transfusion for each patient received on the day of surgery. CABG - coronary artery bypass grafting; $\mathrm{Cl}$ - confidence interval; CSAKI - cardiac surgery associated acute kidney injury; CPB — cardiopulmonary bypass; eGFR - estimated glomerular filtration rate, calculated by CKD-EPI formulae; NYHA — New York Heart Association; $\mathrm{OR}$ - odds ratio; RDW — red cell distribution width

Table 3. Subgroup analysis of risk factors for CSAKI.

\begin{tabular}{|c|c|c|c|c|c|c|}
\hline \multirow[t]{2}{*}{ Variables } & \multicolumn{3}{|c|}{ Transfusion } & \multicolumn{3}{|c|}{ Non-transfusion } \\
\hline & OR & $95 \% \mathrm{Cl}$ & $\mathbf{P}$ & OR & $95 \% \mathrm{Cl}$ & $\mathbf{P}$ \\
\hline Male & 2.130 & $1.582-2.866$ & $<0.001$ & 2.216 & $1.722-2.850$ & $<0.001$ \\
\hline Age [years] & 1.030 & $1.016-1.044$ & $<0.001$ & 1.035 & $1.024-1.047$ & $<0.001$ \\
\hline eGFR $<60 \mathrm{~mL} / \mathrm{min} / 1.73 \mathrm{~m}^{2}$ & 1.888 & $1.165-3.060$ & 0.01 & & & \\
\hline CPB time $>120 \mathrm{~min}$ & 2.251 & $1.631-3.107$ & $<0.001$ & 1.675 & $1.257-2.232$ & $<0.001$ \\
\hline NYHA classification 3-4 & 1.384 & $1.004-1.909$ & 0.047 & & & \\
\hline Erythrocyte transfusion [U] ${ }^{*}$ & 1.198 & $1.028-1.391$ & 0.021 & & & \\
\hline Elevated RDW & 1.040 & $0.928-1.167$ & 0.497 (NS) & 1.613 & $1.277-2.037$ & $<0.001$ \\
\hline
\end{tabular}

*The amount of erythrocyte transfusion refers to the total amount of erythrocyte transfusion for each patient received on the day of surgery. $\mathrm{Cl}$ - confidence interval; CSAKI - cardiac surgery associated acute kidney injury; CPB - cardiopulmonary bypass; eGFR - estimated glomerular filtration rate, calculated by CKD-EPI formulae; NYHA - New York Heart Association; OR — odds ratio; RDW - red cell distribution width; NS - not significant 
Table 4. Subgroup analysis of risk factors for CSAKI in patients underwent valve surgery.

\begin{tabular}{|c|c|c|c|c|c|c|}
\hline \multirow[t]{2}{*}{ Variables } & \multicolumn{3}{|c|}{ Transfusion } & \multicolumn{3}{|c|}{ Non-transfusion } \\
\hline & OR & $95 \% \mathrm{Cl}$ & $\mathbf{P}$ & OR & $95 \% \mathrm{Cl}$ & $\mathbf{P}$ \\
\hline Male & 1.035 & $1.020-1.051$ & $<0.001$ & 1.037 & $1.025-1.048$ & $<0.001$ \\
\hline Age [years] & 1.873 & $1.355-2.587$ & $<0.001$ & 2.194 & $1.703-2.826$ & $<0.001$ \\
\hline NYHA classification 3-4 & 1.430 & $1.009-2.026$ & 0.045 & 1.386 & $1.079-1.781$ & 0.011 \\
\hline Erythrocyte transfusion $[U]^{*}$ & 1.194 & $1.006-1.417$ & 0.042 & & & \\
\hline Elevated RDW & & & 0.314 (NS) & 1.877 & $1.470-2.397$ & $<0.001$ \\
\hline
\end{tabular}

*The amount of erythrocyte transfusion refers to the total amount of erythrocyte transfusion for each patient received on the day of surgery. $\mathrm{Cl}$ - confidence interval; CSAKI — cardiac surgery associated acute kidney injury; NYHA — New York Heart Association; OR — odds ratio; RDW - red cell distribution width; NS - not significant

had more comorbidities. The magnitude of elevated RDW and erythrocyte transfusion were significantly higher in those who developed CSAKI as well. In the entire cohort and non-transfused subgroup, the elevated RDW was identified as independent predictor for CSAKI whereas a similar association was not validated in the transfused subgroup.

Although extensive research has been carried out on the prognostic role of RDW, no single study exists which reports transfusion data or includes transfusion patients [9, 15-17]. According to available research, this is the first study describing the effect of transfusion on the prognostic role of RDW in cardiac surgery patients.

Elevated RDW may indicate several pathogeneses during perioperative phases. First, elevated RDW was reported to be associated with systemic inflammatory response and proinflammatory cytokines during CPB surgery [8, 9]. Second, RDW increases when the number of erythrocytes in which hemoglobin is incompletely saturated with oxygen [18]. Finally, an increase of RDW reflects the increase in variation of erythrocyte size caused by oxidative stress [19].

Recent evidence has shown a relationship between RDW and AKI or its outcome [6, 7, 16]. In a previous study, the elevated RDW was indicated as an independent prognostic factor for severity and poor prognosis of CSAKI [7]. However, transfusion characteristics were not reported. The results in the current study showed a consistent interpretation of elevated RDW associating with CSAKI in the entire cohort and non-transfused patients whereas a similar association was not validated in patients receiving erythrocyte transfusion. One possible explanation was that the role of elevated RDW indicating intraoperative inflammatory response and oxidative stress was predominant in non-transfused patients whereas an identical role was inferior as elevated RDW can be attributed to erythrocyte transfusion, which was reported as another predictor for CSAKI [11].

A prior study noted the incremental effect of erythrocyte transfusion on RDW [12]. This elevated RDW was detectable immediately after transfusion and reached its highest value at $24 \mathrm{~h}$ after $\mathrm{RBC}$ transfusion. In the current study, the change of RDW was defined as the difference between RDW measured $24 \mathrm{~h}$ after cardiac surgery and preoperative RDW. If a patient received multiple numbers of $\mathrm{RBC}$ transfusion within the observation period, the cumulative increment of RDW by RBC transfusion will confound its prognostic role. In the transfused subgroup, each unit of erythrocyte transfusion increased $19.8 \%$ the risk of CSAKI. Accordingly, the analogous spectrum of ORs (1.08-1.26) for each unit of transfusion were reported in several studies indicating a solid association between erythrocyte transfusion and AKI [20-23].

\section{Limitations of the study}

There were several limitations in this study. First, it was a single-center retrospective study. Second, hematopoietic factors were not available in the study population, as it was not routinely tested in the cardiac surgery population. Finally, inflammatory cytokines and biomarkers were not measured in the present study. Therefore, the potential association between the severity of inflammation or oxidative stress and elevated RDW was not quantizable.

\section{Conclusions}

In summary, the current study indicated that elevated RDW was associated with the onset of CSAKI in non-transfused cardiac surgery patients. A similar prognostic role of RDW was not valid in 
transfused patients due to the increment effect of transfusion on RDW. This confounding influence shall be considered in further studies evaluating the role of RDW.

\section{Acknowledgements}

We are grateful for the contribution of the study personnel from the department of nephrology, cardiac surgery and critical care and for their for persistent contribution to maintenance of the cardiac surgery database.

\section{Funding}

This work was supported by: 1) Shanghai Clinical Medical Center for Kidney Disease Project support by Shanghai Municipal Health Commission, No. 2017ZZ01015; 2) Shanghai Municipal Hospital Frontier Technology Project supported by Shanghai ShenKang Hospital Development Center No. SHDC12018127; 3) Shanghai "science and technology innovation plan" technical standard project No. 19DZ2205600; 4) Shanghai "science and technology innovation plan" popular science project No. 19DZ2321400; 5) National Natural Science Foundation of China No. 81800677; 6) Youth Fundation of Zhogshan Hospital No. 2020ZSQN44.

\section{Conflicts of interest: None declared}

\section{References}

1. Fuhrman DY, Kellum JA. Epidemiology and pathophysiology of cardiac surgery-associated acute kidney injury. Curr Opin Anaesthesiol. 2017; 30(1): 60-65, doi: 10.1097/ ACO.0000000000000412, indexed in Pubmed: 27820742.

2. Albert C, Haase M, Albert A, et al. Urinary biomarkers may complement the cleveland score for prediction of adverse kidney events after cardiac surgery: a pilot study. Ann Lab Med. 2020; 40(2): 131-141, doi: 10.3343/alm.2020.40.2.131, indexed in Pubmed: 31650729.

3. Kellum JA, Chawla LS. Cell-cycle arrest and acute kidney injury: the light and the dark sides. Nephrol Dial Transplant. 2016; 31(1): 16-22, doi: 10.1093/ndt/gfv130, indexed in Pubmed: 26044835.

4. Koyner JL, Zarbock A, Basu RK, et al. The impact of biomarkers of acute kidney injury on individual patient care. Nephrol Dial Transplant. 2020; 35(8): 1295-1305, doi: 10.1093/ndt/gfz188, indexed in Pubmed: 31725154.

5. Srisawat N, Kellum J. The role of biomarkers in acute kidney injury. Critical Care Clinics. 2020; 36(1): 125-140, doi: 10.1016/j. ccc.2019.08.010.

6. Duchnowski P, Hryniewiecki T, Kuśmierczyk M, et al. Anisocytosis predicts postoperative renal replacement therapy in patients undergoing heart valve surgery. Cardiol J. 2019 [Epub ahead of print], doi: 10.5603/CJ.a2019.0020, indexed in Pubmed: 30799549.

7. Zou Z, Zhuang Y, Liu L, et al. Role of elevated red cell distribution width on acute kidney injury patients after cardiac surgery. BMC Cardiovasc Disord. 2018; 18(1): 166.

8. Pearson TA, Mensah GA, Alexander RW, et al. Markers of inflammation and cardiovascular disease: application to clinical and public health practice: A statement for healthcare professionals from the Centers for Disease Control and Prevention and the American Heart Association. Circulation. 2003; 107(3): 499-511, doi: 10.1161/01.cir.0000052939.59093.45, indexed in Pubmed: 12551878.

9. Förhécz Z, Gombos T, Borgulya G, et al. Red cell distribution width in heart failure: prediction of clinical events and relationship with markers of ineffective erythropoiesis, inflammation, renal function, and nutritional state. Am Heart J. 2009; 158(4): 659-666, doi: 10.1016/j.ahj.2009.07.024, indexed in Pubmed: 19781428.

10. Christen S, Finckh B, Lykkesfeldt J, et al. Oxidative stress precedes peak systemic inflammatory response in pediatric patients undergoing cardiopulmonary bypass operation. Free Radic Biol Med. 2005; 38(10): 1323-1332, doi: 10.1016/j.freeradbiomed.2005.01.016, indexed in Pubmed: 15855050.

11. Karkouti K. Transfusion and risk of acute kidney injury in cardiac surgery. Br J Anaesth. 2012; 109 Suppl 1: i29-i38, doi: 10.1093/ bja/aes422, indexed in Pubmed: 23242748.

12. Spadaro S, Taccone FS, Fogagnolo A, et al. The effects of blood transfusion on red blood cell distribution width in critically ill patients: a pilot study. Transfusion. 2018; 58(8): 1863-1869.

13. Levey AS, Stevens LA, Schmid CH, et al. A new equation to estimate glomerular filtration rate. Ann Intern Med. 2009; 150(9): 604-612, doi: 10.7326/0003-4819-150-9-200905050-00006, indexed in Pubmed: 19414839.

14. Kellum JA, Lameire N. Diagnosis, evaluation, and management of acute kidney injury: a KDIGO summary (Part 1). Crit Care. 2013; 17(1): 204, doi: 10.1186/cc11454, indexed in Pubmed: 23394211.

15. Patel HH, Patel HR, Higgins JM. Modulation of red blood cell population dynamics is a fundamental homeostatic response to disease. Am J Hematol. 2015; 90(5): 422-428.

16. Oh HJ, Park JT, Kim JK, et al. Red blood cell distribution width is an independent predictor of mortality in acute kidney injury patients treated with continuous renal replacement therapy. Nephrol Dial Transplant. 2012; 27(2): 589-594, doi: 10.1093/ndt/ gfr307, indexed in Pubmed: 21712489.

17. Hunziker S, Celi LA, Lee J, et al. Red cell distribution width improves the simplified acute physiology score for risk prediction in unselected critically ill patients. Crit Care. 2012; 16(3): R89, doi: 10.1186/cc11351, indexed in Pubmed: 22607685.

18. Nishiyama $Y$, Niiyama H, Harada $H$, et al. Effect of exercise training on red blood cell distribution width as a marker of impaired exercise tolerance in patients with coronary artery disease. Int Heart J. 2016; 57(5): 553-557, doi: 10.1536/ihj.16-015, indexed in Pubmed: 27581674.

19. Salvagno GL, Sanchis-Gomar F, Picanza A, et al. Red blood cell distribution width: A simple parameter with multiple clinical applications. Crit Rev Clin Lab Sci. 2015; 52(2): 86-105, doi: 10.3109/10408363.2014.992064, indexed in Pubmed: 25535770.

20. Hajjar LA, Vincent JL, Galas FR, et al. Transfusion requirements after cardiac surgery: the TRACS randomized controlled trial. JAMA. 2010; 304(14): 1559-1567, doi: 10.1001/jama.2010.1446, indexed in Pubmed: 20940381.

21. Jiang W, Teng J, Xu J, et al. Dynamic predictive scores for cardiac surgery: associated acute kidney injury. J Am Heart Assoc. 2016; 5(8), doi: 10.1161/JAHA.116.003754, indexed in Pubmed: 27491837.

22. Karkouti K, Wijeysundera DN, Yau TM, et al. Acute kidney injury after cardiac surgery: focus on modifiable risk factors. Circulation. 2009; 119(4): 495-502, doi: 10.1161/CIRCULATIONAHA.108.786913, indexed in Pubmed: 19153273.

23. Parolari A, Pesce LL, Pacini D, et al. Risk factors for perioperative acute kidney injury after adult cardiac surgery: role of perioperative management. Ann Thorac Surg. 2012; 93(2): 584-591, doi: 10.1016/j.athoracsur.2011.09.073, indexed in Pubmed: 22269725 . 